participation. The information pack was introduced in 2010 which may explain why under half reported receiving it. Notably the majority of those that received it found it useful, suggesting that it is an important component of transition. The age that the topic of transition is introduced could be optimised and standardised, as it is widely thought that beginning the transition process early is an essential element for successful transfer to adult care.

\section{G410(P) ARE ADOLESCENTS WITH SICKLE CELL DISEASE SATISFIED WITH THEIR OUT-PATIENT CLINIC EXPERIENCE?}

'S Mohun Kemp, ${ }^{2} \mathrm{H}$ Sivaguru, ${ }^{1} \mathrm{R}$ Crowley, ${ }^{1} \mathrm{DA}$ Yardumian, ${ }^{1} \mathrm{M}$ Roberts-Harewood, ${ }^{1} \mathrm{O}$ Wilkey, ${ }^{1} \mathrm{G}$ Hann. ${ }^{1}$ North Middlesex University Hospital, London, UK; ${ }^{2}$ University College London, London, UK

\subsection{6/archdischild-2015-308599.364}

Aims Adolescents with chronic conditions can prove difficult to engage. Each clinical encounter must be maximised to provide a positive experience that encourages continued attendance and a collaborative relationship between doctor and patient. This project investigated the out-patient experience of adolescents with sickle cell disease.

Methods A questionnaire comprising both qualitative and quantitative questions was distributed to patients aged 13 to 21 years who attended haematology clinics between January and April 2014.

Results 31 adolescent patients completed the questionnaire (response rate of 94\%).

When asked to rate their out-patient experience, the mean score was 6.7/10. Qualitative questioning highlighted themes of dissatisfaction around long waiting times and lack of waiting room activities. There was a wide variation in reported waiting times (see Figure 1). There was a significant relationship between higher waiting times and lower overall out-patient experience score.

Positive comments were made about 'friendly and helpful' health professionals with a rating score of $8.1 / 10$ for staff friendliness.

During the consultation the majority of patients felt they had enough time to discuss their concerns. Older patients were more likely to have the opportunity to speak to the doctor or nurse without a parent present (see Figure 2). Of those who did not have this opportunity, $23 \%$ stated they would like to talk to a doctor alone.

How long do you normally wait to see a doctor?

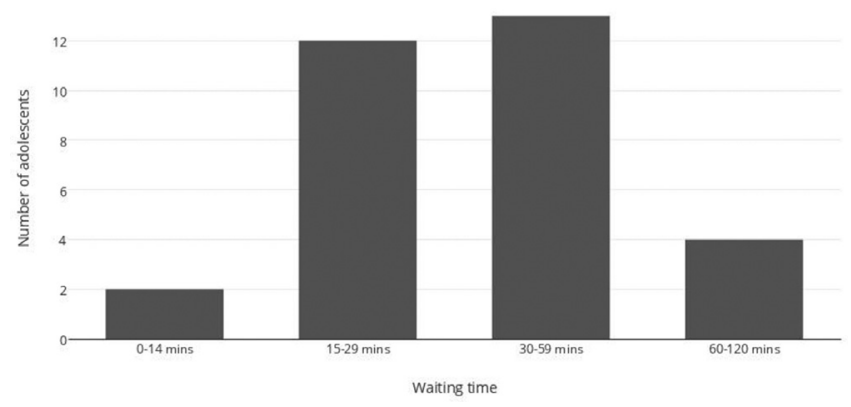

Abstract G410(P) Figure 1 Waiting times
When in clinic, do you have an opportunity to speak to the doctor/nurse without your parents?

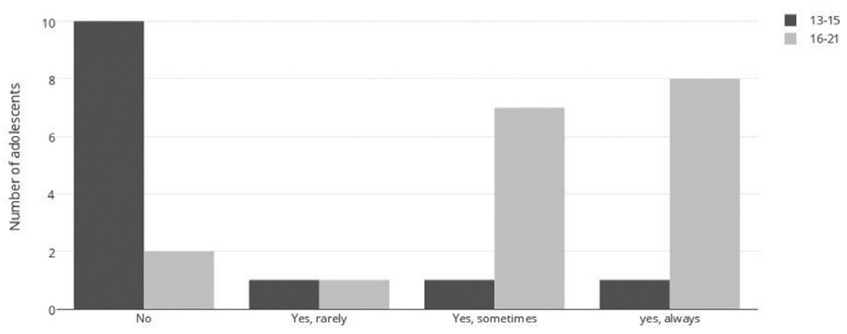

Abstract G410(P) Figure 2 Discussion with doctor

Conclusion There is room to improve the out-patient clinic experience for patients with sickle cell disease. Specifically, reducing waiting times should be given priority. Both clinicians and patients need to arrive promptly to prevent a backlog delay. The clinic environment could be modified to provide a more comfortable and stimulating place for adolescents. Although financial and spatial constraints limit refurbishment, patient feedback suggests simple measures such as installing a water dispenser in the paediatric waiting room may improve patient satisfaction. Finally adolescent patients could be given more opportunity to speak to clinicians without a parent present and this could be introduced at a younger age. However, both our doctors and the adolescents themselves reported that there is often parental resistance to this, which needs to be overcome.

\section{G411(P) A RARE CASE OF PRIMARY ANTIPHOSPHOLIPID SYNDROME}

R Onita, C Reynolds, A Joshi. Luton and Dunstable University Hospital, Luton, UK

10.1136/archdischild-2015-308599.365

Introduction Antiphopholipid syndrome (APS) is a systemic autoimmune condition characterised by venous or arterial thrombosis, hypercoagulability and pregnancy comorbidities in the presence of circulating antibodies directed against phospholipids. Cerebrovascular disease including sinus vein thrombosis and ischaemic stroke are the presenting features in approximately $30 \%$ of cases. Primary APS in the paediatic population is very rare with the exact incidence unknown. A significant number of cases of APS will be associated with autoimmune disease, particularly systemic lupus erythematosus. There are no studies on the management of paediatric APS, which makes the management of these children a challenge for the paediatrician

Case description A 2 year old girl, with no comorbidities presented to the local hospital with one episode of tonic-clonic seizure. Of note, is that for the previous days she became more lethargic, decreased appetite and decreased speech. She was developing normally and there was no family history of note. She had a full septic screen but this showed no evidence of acute infection. An MRI head showed left transverse sinus thrombosis with bilateral thalamic infarcts and right basal ganglia infarction. In view of these findings she had a full thrombophilia screen. The results showed evidence of APS with positive anti cardiolipin antibodies. There was no laboratory features of a secondary autoimmune disease, so a diagnosis of primary APS was made (ANA, ANCA, R. F., C3, C4, IG A, G, M-, Direct Antiglobulin test, PR3, MPO,-negative). She has been anticoagulated with warfarin but her ongoing management has been difficult.

Discussion Evidence for the treatment of APS is based on adult studies and the treatment itself comes with it's own 